\title{
ROUGH PICTURE FUZZY SET AND PICTURE FUZZY TOPOLOGIES
}

\author{
NGUYEN XUAN THAO ${ }^{\dagger}$ AND NGUYEN VAN DINH ${ }^{\ddagger}$ \\ Faculty of Information Technology, Vietnam National University of Agriculture, \\ ${ }^{\dagger}$ thaonx281082@yahoo.com; ${ }^{\ddagger}$ nvdinh2000@gmail.com
}

\begin{abstract}
check
Abstract. Approximation of a picture fuzzy set on a crisp approximation space gives a rough picture fuzzy set. In this paper, the concept of a rough picture set is introduced, besides we also investigate some topological structures of a rough picture fuzzy set are investigated, such are lower and upper rough picture fuzzy approximation operators.
\end{abstract}

Keywords. Rough set, picture fuzzy set, rough picture fuzzy set, approximation operators, picture fuzzy topological space.

\section{INTRODUCTION}

Rough set theory was introduced by Z. Pawlak in 1980s [1]. It becomes a usefully mathematical tool for data mining, especially for redundant and uncertain data. At first, the establishment of the rough set theory is based on equivalence relation. The set of equivalence classes of the universal set, obtained by an equivalence relation, is the basis for the construction of upper and lower approximation of the subset of the universal set.

Fuzzy set theory was introduced by L. Zadeh since 1965 [2]. Immediately, it became a useful method to study the problems of imprecision and uncertainty. After that, there are some extensions of fuzzy set, which also widely used. Intuitionistic fuzzy sets were introduced in 1986, by K. Atanassov [3], which is a generalization of the notion of a fuzzy set. In 2013, B. C. Cuong and V. Kreinovich introduced the concept of picture fuzzy set [4], in which a given set were to be in with three memberships: a degree of positive membership, a degree of negative membership, and a degree of neutral membership of an element in this set. After that, L. H. Son gives an application of picture fuzzy set in the problems of clustering [5].

In addition, combining rough set and fuzzy set has also many interesting results. The approximation of rough (or fuzzy) sets in fuzzy approximation space gives us the fuzzy rough set [6-8]; and the approximation of fuzzy sets in crisp approximation space gives us the rough fuzzy set $[6,7]$. W.Z. $\mathrm{Wu}$ et al. [8] present a general framework for the study of fuzzy rough sets in both constructive and axiomatic approaches. By the same, W. Z. Wu et al. [9] studied the fuzzy topological structures on the rough fuzzy sets, in which both constructive and axiomatic approaches were used. In 2012, Y. $\mathrm{H}$. Xu and W. Z. Wu also investigated the rough intuitionistic fuzzy set and the intuitionistic fuzzy topologies in crisp approximation spaces [10]. Recently, the researchers combine T-rough set with fuzzy set and obtain the set, named "T-rough fuzzy set" [11], which are the generalized rough fuzzy sets.

In this paper, the concept of rough picture fuzzy set is introduced, this is the approximation of picture fuzzy set in crisp approximation space, and some properties of rough picture fuzzy sets are 
under study. After that, "we investigate some topological structures of rough picture fuzzy sets". The paper is organized as following: Section 2 recalls basic notions of picture fuzzy set and rough set. Section 3 introduces rough picture fuzzy set on the crisp approximation space. Section 4 introduces the basic concepts of picture fuzzy topological spaces. Finally, the picture fuzzy topologies of rough picture fuzzy sets are investigated.

\section{BASIC NOTIONS OF PICTURE FUZZY SET AND ROUGH SET}

In this paper, $U$ denotes a nonempty set called the universe of discourse. $P(U)$ will denote the collection of all subsets of $U$ will be denoted by

Definition $1([4])$. A picture fuzzy set $A$ on the universe $U$ is an object of the form

$$
A=\left\{\left(x, \mu_{A}(x), \gamma_{A}(x), \eta_{A}(x)\right) \mid x \in U\right\}
$$

where $\mu_{A}(x)(\in[0,1])$ is called the "degree of positive membership of $x$ in $A^{\prime \prime}, \gamma_{A}(x)(\in[0,1])$ called the "degree of negative membership of $x$ in $A^{\prime \prime}$ and $\eta_{A}(x)(\in[0,1])$ called the "degree of neutral membership of $x$ in $A^{\prime \prime}$, and where $\mu_{A}, \gamma_{A}$ and $\eta_{A}$ satisfy the following condition:

$$
\mu_{A}(x)+\gamma_{A}(x)+\eta(x) \leq 1,(\forall x \in U) .
$$

The family of all picture fuzzy set in $U$ is denoted by $P F S(U)$. The complement of a picture fuzzy set $A$ is denoted by $\sim A=\left\{\left(x, \gamma_{A}(x), \mu_{A}(x), \eta_{A}(x)\right) \mid \forall x \in U\right\}$.

Formally, a picture fuzzy set associate three fuzzy sets $x, \gamma_{A}(x), \mu_{A}(x), \eta_{A}(x) \mu_{A}: U \rightarrow[0,1], \gamma_{A}:$ $U \rightarrow[0,1]$ and $\eta_{A}: U \rightarrow[0,1]$ and $\mu_{A}(x)+\gamma_{A}(x)+\eta(x) \leq 1,(\forall x \in U)$. Obviously, any intuitionistic fuzzy set $A=\left\{\left(x, \mu_{A}(x), \gamma_{A}(x)\right)\right\}$ may be identified with the picture fuzzy set in the form $A=\left\{\left(x, \mu_{A}(x), \gamma_{A}(x), 0\right) \mid x \in U\right\}$.

The operators on $P F S(U)$ are introduced [1]: $\forall A, B \in P F S(U)$,

- $A \subseteq B$ iff $\mu_{A}(x) \leq \mu_{B}(x), \gamma_{A}(x) \geq \gamma_{B}(x)$ and $\eta_{A}(x) \leq \eta_{B}(x) \forall x \in U$,

- $A=B$ iff $A \subseteq B$ and $B \subseteq A$,

- $A \cup B=\left\{\left(x, \max \left(\mu_{A}(x), \mu_{B}(x)\right), \min \left(\gamma_{A}(x), \gamma_{B}(x)\right), \min \left(\eta_{A}(x), \eta_{B}(x)\right) \mid x \in U\right\}\right.$,

- $A \cap B=\left\{\left(x, \min \left(\mu_{A}(x), \mu_{B}(x)\right), \max \left(\gamma_{A}(x), \gamma_{B}(x)\right), \min \left(\eta_{A}(x), \eta_{B}(x)\right) \mid x \in U\right\}\right.$

Now, some special picture fuzzy sets are defined: a constant picture fuzzy set is the picture fuzzy set $(\widehat{\alpha, \beta, \theta})=\{(x, \alpha, \beta, \theta) \mid x \in U\}$; the picture fuzzy universe set is $U=1_{U}=(\widehat{1,0,0})=$ $\{(x, 1,0,0) \mid x \in U\}$ and the picture fuzzy empty set is $\left.\emptyset=0_{U}=\widehat{(0,1,0}\right)=\{(x, 0,1,0) \mid x \in U\}$.

For any $x \in U$, picture fuzzy sets $1_{x}$ and $1_{U-\{x\}}$ are, respectively, defined by: for all $y \in U$

$$
\begin{gathered}
\mu_{1_{x}}(y)=\left\{\begin{array}{ll}
1 & \text { if } y=x \\
0 & \text { if } y \neq x
\end{array}, \quad \gamma_{1_{x}}(y)=\left\{\begin{array}{ll}
0 & \text { if } y=x \\
1 & \text { if } y \neq x
\end{array}, \quad \eta_{1_{x}}(y)= \begin{cases}0 & \text { if } y=x \\
0 & \text { if } y \neq x\end{cases} \right.\right. \\
\mu_{1_{U-\{x\}}}(y)=\left\{\begin{array}{ll}
0 & \text { if } y=x \\
1 & \text { if } y \neq x
\end{array}, \quad \gamma_{1_{U-\{x\}}}(y)=\left\{\begin{array}{ll}
1 & \text { if } y=x \\
0 & \text { if } y \neq x
\end{array}, \quad \eta_{1_{U-\{x\}}}(y)= \begin{cases}0, & \text { if } y=x \\
0, & \text { if } y \neq x\end{cases} \right.\right.
\end{gathered}
$$


Definition 2. Let $U$ be a nonempty universe of discourse which may be infinite. A subset $R \in$ $P(U \times U)$ is referred to as a (crisp) binary relation on $U$. The relation $R$ is referred to as:

- Reflexive: if for all $x \in U,(x, x) \in R$

- Symmetric: if for all $x, y \in U,(x, y) \in R$ then $(y, x) \in R$

- Transitive: if for all $x, y, z \in U,(x, y) \in R,(y, z) \in R$ then $(x, z) \in R$

- Similarity: if $R$ is reflexive and symmetric,

- Preorder: if $R$ is reflexive and transitive,

- Equivalence: if $R$ is reflexive and symmetric, transitive.

A crisp approximation space is a pair $(U, R)$ For an arbitrary crisp relation $R$ on $U$, a set-valued mapping $R_{s}: U \rightarrow P(U)$ can be defined by:

$$
R_{s}(x)=\{y \in U \mid(x, y) \in R\}, x \in U .
$$

Then, $R_{s}(x)$ is called the successor neighborhood of $x$ with respect to (w.r.t) $R$.

Definition 3 ([1]). Let $(U, R)$ be a crisp approximation space. For each $A \subseteq U$, the upper and lower approximations of $A$ (w.r.t) $(U, R)$ denoted by $R^{\prime \prime}$ and $R^{\prime \prime}$, respectively, are defined as follows

$$
\begin{aligned}
& \bar{R}(A)=\left\{x \in U: R_{s}(X) \cap A \neq \emptyset\right\} \\
& \underline{R}(A)=\left\{x \in U: R_{s}(x) \subseteq A\right\}
\end{aligned}
$$

\section{ROUGH PICTURE FUZZY SET}

A rough picture fuzzy set is the approximation of a picture fuzzy set w.r.t a crisp approximation space. Here, the upper and lower approximations of a picture fuzzy set in the crisp approximation spaces together with their membership functions, respectively are considered

Definition 4. Let $(U, R)$ be a crisp approximation space. For $A \in P F S(U)$, the upper and lower approximations of $A$ (w.r.t) $(U, R)$ denoted by $\overline{R P}(A)$ and $\underline{R P}(A)$, respectively, are defined as follows:

$$
\begin{aligned}
& \overline{R P}(A)=\left\{\left(x, \mu_{\overline{R P}(A)}(x), \gamma_{\overline{R P}(A)}(x), \eta_{\overline{R P}(A)}(x)\right) \mid x \in U\right\}, \\
& \underline{R P}(A)=\left\{\left(x, \mu_{\underline{R P}(A)}(x), \gamma_{\underline{R P}(A)}(x), \eta_{\underline{R P}(A)}(x)\right) \mid x \in U\right\}
\end{aligned}
$$

where

$$
\begin{array}{lll}
\mu_{\overline{R P}(A)}(x)=\vee_{y \in R_{s}(x)} \mu_{A}(y), & \gamma_{\overline{R P}(A)}(x)=\wedge_{y \in R_{s}(x)} \gamma_{A}(y), & \eta_{\overline{R P}(A)}(x)=\wedge_{y \in R_{s}(x)} \eta_{A}(y), \\
\mu_{\underline{R P}(A)}(x)=\wedge_{y \in R_{s}(x)} \mu_{A}(y), & \gamma_{\underline{R P}(A)}(x)=\vee_{y \in R_{s}(x)} \gamma_{A}(y), & \eta_{\underline{R P}(A)}(x)=\wedge_{y \in R_{s}(x)} \eta_{A}(y) .
\end{array}
$$

It is easy to verify that $\overline{R P}(A)$ and $\underline{R P}(A)$ are two picture fuzzy sets in $U$ thus picture mappings $\overline{R P}, \underline{R P}: P F S(U) \rightarrow P F S(U)$ are referred to as the upper and lower picture fuzzy approximation operators, respectively, and the pair $(\underline{R P}(A), \overline{R P}(A))$ is called the rough picture fuzzy set of $A$ w.r.t the approximation space $(U, R)$. 
Example 1. Let $U=\left\{u_{1}, u_{2}, \ldots, u_{10}\right\}$ be a universe set and $R$ be an equivalence relation on $U$, in which the equivalence classes of each element in $U$ defined by $R$ as follows:

$$
U / R=\left\{X_{1}=\left\{u_{1}, u_{3}, u_{9}\right\}, X_{2}=\left\{u_{2}, u_{7}, u_{10}\right\}, X_{3}=\left\{u_{4}\right\}, X_{4}=\left\{u_{5}, u_{8}\right\}, X_{5}=\left\{u_{6}\right\}\right\} .
$$

For a picture fuzzy set $A=\frac{(0.2,0.5,0.3)}{u_{1}}+\frac{(0.3,0.5,0.1)}{u_{2}}+\frac{(0.6,0.4,0)}{u_{3}}+\frac{(0.15,0.7,0.1)}{u_{4}}+\frac{(0.05,0.7,0.2)}{u_{5}}$ $+\frac{(0.1,0.5,0.3)}{u_{6}}+\frac{(0.25,0.4,0.3)}{u_{7}}+\frac{(0.1,0.2,0.6)}{u_{8}}+\frac{(0.45,0.45,0.1)}{u_{9}}+\frac{(0.05,0.9,0.05)}{u_{10}}$ of $U$.

We have

$\mu_{\overline{R P}(A)}\left(u_{i}\right)=\vee_{y \in R_{s}\left(u_{i}\right)} \mu_{A}(y)=\vee_{y \in X_{1}} \mu_{A}(y)=0.6$, for all $u_{i} \in X_{1}$,

$\mu_{\underline{R P}(A)}\left(u_{i}\right)=\wedge_{y \in R_{s}\left(u_{i}\right)} \mu_{A}(y)=\wedge_{y \in X_{1}} \mu_{A}(y)=0.2$, for all $u_{i} \in X_{1}$,

$\gamma_{\overline{R P}(A)}\left(u_{i}\right)=\wedge_{y \in R_{s}\left(u_{i}\right)} \gamma_{A}(y)=\wedge_{y \in X_{1}} \gamma_{A}(y)=0.4$, for all $u_{i} \in X_{1}$,

$\gamma_{R P(A)}\left(u_{i}\right)=\vee_{y \in R_{s}\left(u_{i}\right)} \gamma_{A}(y)=\vee_{y \in X_{1}} \gamma_{A}(y)=0.5$ for all $u_{i} \in X_{1}$,

$\eta_{\overline{R P}(A)}\left(u_{i}\right)=\wedge_{y \in R_{s}\left(u_{i}\right)} \eta_{A}(y)=\wedge_{y \in X_{1}} \eta_{A}(y)=0$, for all $u_{i} \in X_{1}$,

$\eta_{R P(A)}\left(u_{i}\right)=\wedge_{y \in R_{s}\left(u_{i}\right)} \eta_{A}(y)=\wedge_{y \in X_{1}} \eta_{A}(y)=0$ for all $u_{i} \in X_{1}$,

for all $u_{i} \in X_{2}, \mu_{\overline{R P}(A)}\left(u_{i}\right)=0.3, \mu_{\underline{R P}(A)}\left(u_{i}\right)=0.05, \gamma_{\overline{R P}(A)}\left(u_{i}\right)=0.4, \gamma_{\underline{R P}(A)}\left(u_{i}\right)=0.9$, $\eta_{\overline{R P}(A)}\left(u_{i}\right)=0.05, \eta_{\underline{R P}(A)}\left(u_{i}\right)=0.05$,

for all $u_{i} \in X_{3}, \mu_{\overline{R P}(A)}\left(u_{i}\right)=0.15, \mu_{\underline{R P}(A)}\left(u_{i}\right)=0.15, \gamma_{\overline{R P}(A)}\left(u_{i}\right)=0.7, \gamma_{\underline{R P}(A)}\left(u_{i}\right)=0.7$, $\eta_{\overline{R P}(A)}\left(u_{i}\right)=0.1, \eta_{\underline{R P}(A)}\left(u_{i}\right)=0.1$,

for all $u_{i} \in X_{4}, \mu_{\overline{R P}(A)}\left(u_{i}\right)=0.1, \mu_{\underline{R P}(A)}\left(u_{i}\right)=0.05, \gamma_{\overline{R P}(A)}\left(u_{i}\right)=0.2, \gamma_{\underline{R P}(A)}\left(u_{i}\right)=0.7$, $\eta_{\overline{R P}(A)}\left(u_{i}\right)=0.2, \eta_{\underline{R P}(A)}\left(u_{i}\right)=0.2$,

for all $u_{i} \in X_{5}, \mu_{\overline{R P}(A)}\left(u_{i}\right)=0.1, \mu_{\underline{R P}(A)}\left(u_{i}\right)=0.1, \gamma_{\overline{R P}(A)}\left(u_{i}\right)=0.5, \gamma_{\underline{R P}(A)}\left(u_{i}\right)=0.5$, $\eta_{\overline{R P}(A)}\left(u_{i}\right)=0.05, \eta_{\underline{R P}(A)}\left(u_{i}\right)=0.05$.

So that, the upper approximation picture fuzzy set of the picture fuzzy set $A$ is $\overline{R P}(A)=$ $\frac{(0.6,0.4,0)}{u_{1}}+\frac{(0.3,0.4,0.05)}{u_{2}}+\frac{(0.6,0.4,0)}{u_{3}}+\frac{(0.15,0.7,0.1)}{u_{4}}+\frac{(0.1,0.2,0.2)}{u_{5}}+\frac{(0.1,0.5,0.3)}{u_{6}}+\frac{(0.3,0.4,0.05)}{u_{7}}+\frac{(0.1,0.2,0.2)}{u_{8}}+$ $\frac{(0.6,0.4,0)}{u_{9}}+\frac{(0.3,0.4,0.05)}{u_{10}}$, and the upper approximation picture fuzzy set of the picture fuzzy set $A$

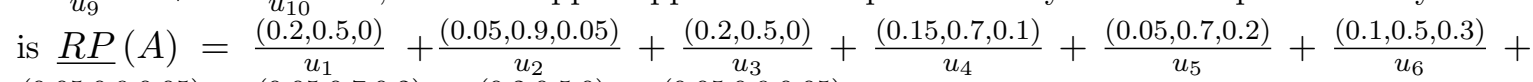
$\frac{(0.05,0.9,0.05)}{u_{7}}+\frac{(0.05,0.7,0.2)}{u_{8}}+\frac{(0.2,0.5,0)}{u_{9}}+\frac{(0.05,0.9,0.05)}{u_{10}}$.

They can expressed in forms $\frac{u_{9}}{R P}(A)=\frac{(0.6,0.4,0)}{X_{1}}+\frac{(0.3,0.4,0.05)}{X_{2}}+\frac{(0.15,0.7,0.1)}{X_{3}}+\frac{(0.1,0.2,0.2)}{X_{4}}+$

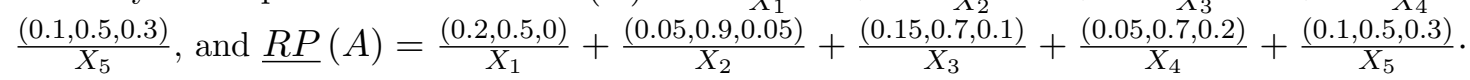

Some basic properties of rough picture fuzzy set approximation operators are listed in following theorems:

Theorem 1. Let $(U, R)$ be a crisp approximation space, then the upper and lower rough picture fuzzy approximation operators defined in Definition 3 satisfy the following properties: $\forall A, B, A_{j} \in \operatorname{PFS}(U), j \in J, J$ is an index set,

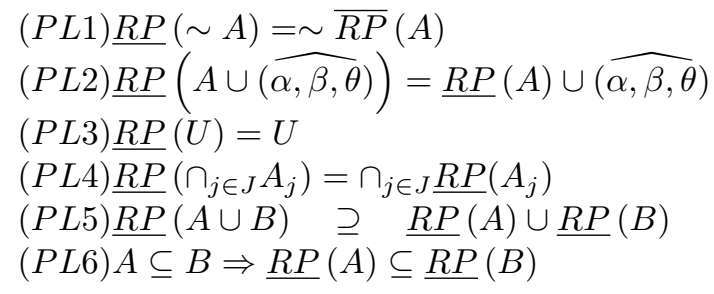

From Definition 3 and Theorem 1, results in
$(P U 1) \overline{R P}(\sim A)=\sim \underline{R P}(A)$

$(P U 2) \overline{P R}(A \cap(\widehat{\alpha, \beta, \theta}))=\overline{P R}(A) \cap(\widehat{\alpha, \beta, \theta})$

$(P U 3) \overline{P R}(\emptyset)=\emptyset$

$(P U 4) \overline{R P}\left(\cup_{j \in J} A_{j}\right)=\cup_{j \in J} \overline{R P}\left(A_{j}\right)$

$(P U 5) \overline{R P}(A \cap B) \subseteq \overline{R P}(A) \cap \overline{R P}(B)$

$(P U 6) A \subseteq B \Rightarrow \overline{R P}(A) \subseteq \overline{R P}(B)$ 
Theorem 2. Let $(U, R)$ be a crisp approximation space. Then

$\underline{R P}(U)=U=\overline{R P}(U)$ and $\underline{R P}(\emptyset)=\emptyset=\overline{R P}(\emptyset)$.

$\underline{R P}(A) \subseteq \overline{R P}(A)$ for all $A \in P F S(U)$

In the case of connections between special types of crisp relation on $U$, and properties of rough picture fuzzy approximation operators yield.

Lemma 1. If $R$ is a symmetric crisp binary relation on $U$, then for all $A, B \in P F S(U)$

$$
\overline{R P}(A) \subseteq B \Leftrightarrow A \subseteq \underline{R P}(B)
$$

Proof. Let $R$ be a symmetric crisp binary relation on $U$, i.e, $y \in R_{s}(x) \Leftrightarrow x \in R_{s}(y) \forall x, y \in$ $U$. The researchers assume contradiction that $\overline{R P}(A) \subseteq B$ but $A \underline{R P}(B)$. For each $x \in$ $U$, all the cases are considered: If $\mu_{A}(x)>\mu_{\underline{R P}(B)}(x)=\wedge_{y \in R_{s}(x)} \mu_{B}(y)$ then it exists $y^{\prime} \in R_{s}(x)$ such that $\mu_{A}(x)>\mu_{B}\left(y^{\prime}\right) \geq \mu_{\overline{R P}(A)}\left(y^{\prime}\right)=\vee_{z \in R_{s}\left(y^{\prime}\right)} \mu_{A}(z) \geq \mu_{A}(x)$, because $\left.x \in R_{s}\left(y^{\prime \prime}\right)\right)$ This is not true. If $\gamma_{A}(x)<\gamma_{R P B}(x)=\vee_{y \in R_{S}(x)} \gamma_{B}(y)$, then it exists $y^{\prime} \in R_{s}(x)$ such that $\gamma_{B}\left(y^{\prime}\right)>\gamma_{A}(x) \geq \wedge_{\left.x \in R_{s}\left(y^{\prime \prime}\right)\right)}$, this is also not true. Similarly, it is false, if $\eta_{A}(x)>\eta_{R P(B)}(x)$. Hence $\overline{R P}(A) \subseteq B \Rightarrow A \subseteq \underline{R P}(B)$. By the same way, it yields $A \subseteq \underline{R P}(B) \Rightarrow \overline{R P}(A) \subseteq B$.

Theorem 3. Let $(U, R)$ be a crisp approximation space, and $\overline{R P}, \underline{R P}: \operatorname{PFS}(U) \rightarrow \operatorname{PFS}(U)$ are the upper and lower picture fuzzy approximation operators. Then

$$
\begin{aligned}
& R \text { is reflexive } \quad \Leftrightarrow(P L R) \underline{R P}(A) \subseteq A \forall A \in P F S(U) \\
& \Leftrightarrow(P U R) \overline{A \subseteq} \overline{R P}(A) \forall A \in P F S(U) \\
& R \text { is symmetric } \Leftrightarrow(P L R) \overline{R P}(\underline{R P}(A)) \subseteq A \forall A \in P F S(U) \\
& \Leftrightarrow(P U R) A \subseteq \underline{R P}(\overline{R P}(A)) \forall A \in P F S(U) \\
& \Leftrightarrow(P L S)^{\prime} \mu_{\underline{R P}\left(1_{U-\{x\}}\right)}(y)=\mu_{\underline{R P}\left(1_{U-\{y\}}\right)}(x) \forall x, y \in U \\
& \Leftrightarrow(P U S)^{\prime} \mu_{\overline{R P}\left(1_{x}\right)}(y)=\mu_{\overline{R P}\left(1_{y}\right)}(x) \forall x, y \in U \\
& \Leftrightarrow(P L S)^{\prime \prime} \gamma_{\underline{R P}\left(1_{U-\{x\}}\right)}(y)=\gamma_{\underline{R P}\left(1_{U-\{y\}}\right)}(x) \forall x, y \in U \\
& \Leftrightarrow(P U S)^{\prime \prime} \gamma_{\overline{R P}\left(1_{x}\right)}(y)=\gamma_{\overline{R P}\left(1_{y}\right)}(x) \forall x, y \in U \text {. } \\
& R \text { is transitive } \Leftrightarrow(P L T) \underline{R P}(A) \subseteq \underline{R P}(\underline{R P}(A)) \forall A \in P F S(U) \\
& \Leftrightarrow(P U T) \overline{\overline{R P}}(A) \subseteq \overline{\overline{R P}} \overline{(\overline{R P}}(A)) \forall A \in P F S(U)
\end{aligned}
$$

Now, according to Theorem 1, Lemma 1 and Theorem 3, it results in the following:

Theorem 4. Let $R$ be a similarity crisp binary relation on $U$ and $\overline{R P}, \underline{R P}: \operatorname{PFS}(U) \rightarrow$ $P F S(U)$ are the upper and lower PF approximation operators. Then, for all $A \in P F S(U)$

$$
A=\underline{R P}(A) \Leftrightarrow \overline{R P}(A)=A \Leftrightarrow \sim A=\underline{R P}(\sim A) \Leftrightarrow \overline{R P}(\sim A)=\sim A
$$

\section{BASIC CONCEPTS OF PICTURE FUZZY TOPOLOGICAL SPACES}

In this section, basic concepts relating to picture fuzzy topological spaces are introduced.

Definition 5. A picture fuzzy topology in the sense of Lowen [12] on a nonempty set $U$ is a family $\tau$ of picture fuzzy sets in $U$ satisfying the following axioms:

(T1) $(\widehat{\alpha, \beta, \theta}) \in \tau$ for all $(\widehat{\alpha, \beta, \theta}) \in \operatorname{PFS}(\mathrm{U})$

(T2) $G_{1} \cap G_{2} \in \tau$ for any $G_{1}, G_{2} \in \tau$

(T3) $\cup_{i \in I} G_{i} \in \tau$ for a family $\left\{G_{i} \mid i \in I\right\} \subseteq \tau$, where $I$ is an index set. 
In this case the pair $(U, \tau)$ is called a picture fuzzy topological space and each picture fuzzy set $A$ in $\tau$ is referred to as a picture fuzzy open set in $(U, \tau)$. The complement of a picture fuzzy set in the picture fuzzy topological space $(U, \tau)$ is called a picture fuzzy closed set in $(U, \tau)$.

Now, the picture fuzzy closure and interior operations in a picture fuzzy topological space are defined.

Definition 6. Let $(U, \tau)$ be a picture fuzzy topological space and $A \in P F S(U)$. Then the picture fuzzy interior and picture fuzzy closure of $A$ are, respectively, defined as follows:

$$
\begin{aligned}
\operatorname{int}(A) & =\cup\{G \mid G \text { is a picture fuzzy open set and } G \subseteq A\} \\
\operatorname{cl}(A) & =\cap\{K \mid K \text { is a picture fuzzy closed set and } A \subseteq K\}
\end{aligned}
$$

and int, cl $: P F S(U) \rightarrow P F S(U)$ are, respectively, called the picture fuzzy interior operator and the picture fuzzy closure operator of $\tau$, and sometimes in order to distinguish, we denote them by int $_{\tau}$ and $c l_{\tau}$

Clearly, int $(A)$ and $c l(A)$ are a picture fuzzy open set and closed set in $(U, \tau)$, respectively, and

$A$ is a picture fuzzy open set in $(U, \tau)$ iff $\operatorname{int}(A)=A$

$A$ is a picture fuzzy closed set in $(U, \tau)$ iff $\operatorname{cl}(A)=A$

Note that $\operatorname{int}(\sim A)=\sim \operatorname{cl}(A)$ and $\operatorname{cl}(\sim A)=\operatorname{int}(\sim A)$ for all $A \in P F S(U)$.

It is easy to show that the picture fuzzy interior operator satisfies following properties:

(Int1) int $((\widehat{\alpha, \beta, \theta}))=(\widehat{\alpha, \beta, \theta})$, for all $(\widehat{\alpha, \beta, \theta}) \in P F S(U)$,

(Int2) $\operatorname{int}(A \cap B)=\operatorname{int}(A) \cap \operatorname{int}(B), \forall A, B \in P F S(U)$,

(Int3) $\operatorname{int}(\operatorname{int}(A))=\operatorname{int}(A), \forall A \in P F S(U)$,

(Int4) $\operatorname{int}(A) \subseteq A, \forall A \in P F S(U)$.

Properties (Int1)-(Int4) are called the picture fuzzy open axioms.

Similarly, the PF closure operator satisfies following properties:

(Cl1) $c l((\widehat{\alpha, \beta, \theta}))=(\widehat{\alpha, \beta, \theta})$, for all $(\widehat{\alpha, \beta, \theta}) \in P F S(U)$

$(\mathrm{Cl} 2) \operatorname{cl}(A \cup B)=\operatorname{cl}(A) \cup \operatorname{cl}(B), \forall A, B \in P F S(U)$

(Cl3) $\operatorname{cl}(\operatorname{cl}(A))=\operatorname{cl}(A),, \forall A \in P F S(U)$

(Cl4) $A \subseteq \operatorname{cl}(A), \forall A \in P F S(U)$

\section{Definition 7.}

(i) A mapping int : $P F S(U) \rightarrow P F S(U)$ is referred to as a fuzzy interior operator if it satisfies axioms (Int1)-(Int4).

(ii) A mapping $c l: P F S(U) \rightarrow P F S(U)$ is referred to as a fuzzy closure operator if it satisfies axioms (Cl1)-(Cl4)

It can be verified that a picture fuzzy interior operator int defined in Definition 7 (i) determines a picture fuzzy topology

$$
\tau_{\text {int }}=\{A \in \operatorname{PFS}(U) \mid \operatorname{int}(A)=A\}
$$

Dually, from a picture fuzzy closure operator cl defined in Definition 7 (ii), it yields a picture fuzzy topology on $U$ by setting

$$
\tau_{c l}=\{A \in \operatorname{PFS}(U) \mid c l(\sim A)=\sim A\}
$$




\section{PICTURE FUZZY TOPOLOGIES OF ROUGH PICTURE FUZZY SETS}

In this section, the relationship between picture fuzzy topological spaces and rough picture fuzzy sets is under discussion. Let $(U, R)$ be a crisp approximation space, and $\overline{R P}, \underline{R P}: P F S(U) \rightarrow P F S(U)$ are the upper and lower picture fuzzy approximation operators which defined in Definition 3.

We denote $\tau_{R}^{\prime \prime}=\{A \in P F S(U) \underline{\mid R P}(A)=\overline{R P}(A)\}$.

Theorem 5. If $R$ is a crisp binary relation on $U$, then $\tau_{R}^{\prime \prime}$ will be a picture fuzzy topology on $U$.

Proof. Theorem 2 shows that $\emptyset, U \in \tau_{R}^{\prime \prime}$. Now, all the conditions of Definition 5 for $\tau_{R}^{\prime \prime}$ are considered.

(T1) Given $(\widehat{\alpha, \beta, \theta}) \in \operatorname{PFS}(U)$. Then, for all $x \in U$

$$
\begin{aligned}
-\mu_{\underline{R P}(\widehat{\alpha, \beta, \theta})}(x) & =\wedge_{y \in R_{s}(x)} \mu_{(\widehat{\alpha, \beta, \theta})}(y)=\wedge_{y \in R_{s}(x)} \alpha=\alpha \\
& =\mu_{\overline{R P}(\widehat{\alpha, \beta, \theta})}(x)=\vee_{y \in R_{s}(x)} \mu_{(\widehat{\alpha, \beta, \theta})}(y)=\vee_{y \in R_{s}(x)} \alpha, \\
\text { - } \gamma_{\underline{R P}(\widehat{\alpha, \beta, \theta})}(x) & =\vee_{y \in R_{s}(x)} \gamma_{(\widehat{\alpha, \beta, \theta})}(y)=\vee_{y \in R_{s}(x)} \beta=\beta \\
& =\gamma_{\overline{R P}(\widehat{\alpha, \beta, \theta})}(x)=\wedge_{y \in R_{s}(x)} \gamma_{(\widehat{\alpha, \beta, \theta})}(y)=\wedge_{y \in R_{s}(x)} \beta, \\
\text { - } \eta_{\underline{R P}(\widehat{\alpha, \beta, \theta})}(x) & =\wedge_{y \in R_{s}(x)} \eta_{(\widehat{\alpha, \beta, \theta})}(y)=\wedge_{y \in R_{s}(x)} \theta=\theta=\eta_{\widehat{R P}(\widehat{\alpha, \beta, \theta})}(x) \\
& =\vee_{y \in R_{s}(x)} \eta_{(\widehat{\alpha, \beta, \theta})}(y)=\vee_{y \in R_{s}(x)} \theta .
\end{aligned}
$$

Hence $\underline{R P}(\widehat{\alpha, \beta, \theta})=(\widehat{\alpha, \beta, \theta})=\overline{R P}(\widehat{\alpha, \beta, \theta})$ So $(\widehat{\alpha, \beta, \theta}) \in \tau_{R}^{\prime \prime}$

(T2) If $G_{1}, G_{2} \in \tau_{R}^{\prime \prime}$. , then $\underline{R P}\left(G_{1} \cap G_{2}\right) \subseteq \overline{R P}\left(G_{1} \cap G_{2}\right)$ (Theorem 2) Also, if $G_{1}, G_{2} \in$ $\tau_{R}^{\prime \prime}$, then $\overline{R P}\left(G_{1} \cap G_{2}\right) \subseteq \overline{R P}\left(\overline{\left.G_{1}\right)} \cap \overline{R P}\left(G_{2}\right)=\underline{R P}\left(G_{1}\right) \cap \underline{R P}\left(G_{2}\right)=\underline{R P}\left(G_{1} \cap G_{2}\right)\right.$. Hence $\underline{R P}\left(G_{1} \cap G_{2}\right)=\overline{R P}\left(G_{1} \cap G_{2}\right)$. So that $G_{1} \cap G_{2} \in \tau_{R}^{\prime \prime}$.

(T3) For a family $\left\{G_{i} \mid i \in I\right\} \subseteq \tau^{\prime \prime}{ }_{R} \subset P F S(U)$, then $\underline{R P}\left(\cup_{i \in I} G_{i}\right) \subseteq \overline{R P}\left(\cup_{i \in I} G_{i}\right)$ (Theorem 2). According to Theorem 1, if $\left\{G_{i} \mid i \in I\right\} \subseteq \tau^{\prime \prime}{ }_{R} \subset P F S(U)$ then $\overline{R P}\left(\cup_{i \in I} G_{i}\right)=$ $\cup_{i \in I} \overline{R P}\left(G_{i}\right)=\cup_{i \in I} \underline{R P}\left(G_{i}\right) \subseteq \underline{R P}\left(\cup_{i \in I} G_{i}\right)$. These show that $\underline{R P}\left(\cup_{i \in I} G_{i}\right)=\overline{R P}\left(\cup_{i \in I} G_{i}\right)$. This means $\cup_{i \in I} G_{i} \in \tau_{R}^{\prime \prime}$.

We denote $\tau_{R}=\{A \in \operatorname{PFS}(U) \mid \underline{R P}(A)=A\}$ and $\tau_{R}^{\prime}=\{A \in \operatorname{PFS}(U) \mid \overline{R P}(A)=A\}$. Then, we have:

Theorem 6. If $R$ is a reflexive crisp binary relation on $U$, then $\tau_{R}$ and $\tau_{R}^{\prime}$ will be the picture fuzzy topologies on $U$.

Proof. We check conditions (T1), T(2), (T3) of definition 5 for $\tau_{R}$, and do similary for $\tau_{R}^{\prime}$.

Theorem 2 shows that $\emptyset, U \in \tau_{R}$. Now, all $t$ conditions of Definition 5 for $\tau_{R}$ are considered.

(T1) Given $(\widehat{\alpha, \beta, \theta}) \in P F S(U)$. Then, for all $x \in U$

- $\mu_{\underline{R P}(\widehat{\alpha, \beta, \theta})}(x)=\wedge_{y \in R_{s}(x)} \mu_{(\widehat{\alpha, \beta, \theta})}(y)=\wedge_{y \in R_{s}(x)} \alpha=\alpha$, 
- $\gamma_{\underline{R P}(\widehat{\alpha, \beta, \theta})}(x)=\vee_{y \in R_{s}(x)} \gamma_{(\widehat{\alpha, \beta, \theta})}(y)=\vee_{y \in R_{s}(x)} \beta=\beta$,

- $\eta_{\underline{R P}(\widehat{\alpha, \beta, \theta})}(x)=\wedge_{y \in R_{s}(x)} \eta_{(\widehat{\alpha, \beta, \theta})}(y)=\wedge_{y \in R_{s}(x)} \theta=\theta$.

Hence $(\widehat{\alpha, \beta, \theta}) \in \tau_{R}$.

(T2) Since $R$ is reflexive then $\underline{R P}\left(G_{1} \cap_{2}^{G}\right) \subseteq G_{1} \cap G_{2}$ for all $G_{1}, G_{2} \in \tau_{R} \subset P F S(U)$ (according to Theorem 3). Moreover, since $G_{1}, G_{2} \in \tau_{R}$ then $\underline{R P}\left(G_{1}\right)=G_{1}, \underline{R P}\left(G_{2}\right)=G_{2}$. So that $G_{1} \cap G_{2}=\underline{R P}\left(G_{1}\right) \cap \underline{R P}\left(G_{2}\right) \subseteq \underline{R P}\left(G_{1} \cap G_{2}\right)$ (Theorem 1). Hence $\underline{R P}\left(G_{1} \cap G_{2}\right)=$ $G_{1} \cap G_{2}$. It means $G_{1} \cap G_{2} \in \tau_{R}$ for all $G_{1}, G_{2} \in \tau_{R}$.

(T3) According to Theorem 3, since $R$ is reflexive, then $\underline{R P}\left(\cup_{i \in I} G_{i}\right) \subseteq \cup_{i \in I} G_{i}$ for a family $\left\{G_{i} \mid i \in I\right\} \subseteq \tau_{R} \subset P F S(U)$, where $I$ is an index set. According to Theorem 1, for a family $\left\{G_{i} \mid i \in I\right\} \subseteq \tau_{R} \subset P F S(U)$, then $\cup_{i \in I} G_{i}=\cup_{i \in I} \underline{R P}\left(G_{i}\right) \subseteq \underline{R P}\left(\cup_{i \in I} G_{i}\right)$. Hence $\underline{R P}\left(\cup_{i \in I} G_{i}\right)=\cup_{i \in I} G_{i}$ for a family $\left\{G_{i} \mid i \in I\right\} \subseteq \tau_{R} \subset P F S(U)$. So that $\cup_{i \in I} G_{i} \in \tau_{R}$.

Similar results are confirmed in [10] for intuitionistic fuzzy approximation operator, here similar results for the picture fuzzy approximation operator are also confirmed.

Theorem 6 shows that the lower and upper rough picture fuzzy approximation operators induced from a reflexive and transitive relation are, respectively, a picture fuzzy interior operator and a picture fuzzy closure operator.

Theorem 7. Let $(U, R)$ be a crisp approximation space. Then the following statements are equivalent:

a) $R$ is a reflexive and transitive relation

b) The upper rough picture fuzzy approximation operator $\overline{R P}: P F S(U) \rightarrow P F S(U)$ is a picture fuzzy closure operator,

c) The lower rough picture fuzzy approximation operator $\underline{\operatorname{RP}}: \operatorname{PFS}(U) \rightarrow \operatorname{PFS}(U)$ is a picture fuzzy interior operator

\section{CONCLUSION}

In this paper the concept of rough picture fuzzy set and some properties of it are introduced. Then, the topological structure of rough picture fuzzy sets in corresponding to the properties of a crisp binary set is under study, the lower and upper rough picture fuzzy approximation operators are a picture fuzzy interior operator and a picture fuzzy closure operator respectively if only the crisp binary relation in the crisp approximation space is reflexive and transitive.

\section{REFERENCES}

[1] Z. Pawlak, "Rough sets," International Journal of Computer \& Information Sciences, vol. 11, no. 5, pp. 341-356, 1982.

[2] L. A. Zadeh, "Fuzzy sets," Information and Control, vol. 8, no. 3, pp. 338-353, 1965.

[3] K. T. Atanassov, "Intuitionistic fuzzy sets," Fuzzy Sets and Systems, vol. 20, no. 1, pp. 87-96, 1986. 
[4] B. C. Cuong and V. Kreinovich, "Picture fuzzy sets-a new concept for computational intelligence problems," in Proceedings of the third world congress on information and communication technologies WICT'2013, Hanoi, Vietnam, December 15-18, 2013, pp. 1-6.

[5] L. H. Son, "DPFCM: a novel distributed picture fuzzy clustering method on picture fuzzy sets," Expert Systems with Applications, vol. 42, no. 1, pp. 51-66, 2015.

[6] D. Dubois and H. Prade, "Rough fuzzy sets and fuzzy rough sets*," International Journal of General System, vol. 17, no. 2-3, pp. 191-209, 1990.

[7] Y. Yao, "Combination of rough and fuzzy sets based on $\alpha$-level sets," in Rough sets and Data Mining. Springer, 1997, pp. 301-321.

[8] W.-Z. Wu, J.-S. Mi, and W.-X. Zhang, "Generalized fuzzy rough sets," Information Sciences, vol. 151, pp. 263-282, 2003.

[9] W.-Z. Wu and Y.-H. Xu, "On fuzzy topological structures of rough fuzzy sets," in Transactions on Rough Sets XVI. Springer, 2013, pp. 125-143.

[10] Y.-H. Xu and W.-Z. Wu, "Intuitionistic fuzzy topologies in crisp approximation spaces," in Rough Sets and Knowledge Technology. Springer, 2012, pp. 496-503.

[11] N. V. Dinh, N. X. Thao, and N. M. Chau, "Some properties of generalized rough fuzzy set," in Fundamental and Applied IT Research (FAIR), 2014, pp. 598-601.

[12] R. Lowen, "Fuzzy topological spaces and fuzzy compactness," Journal of Mathematical Analysis and Applications, vol. 56, no. 3, pp. 621-633, 1976.

Received on October $04-2014$

Revised on August 31 - 2015 